\title{
Brachymetatarsia: One-Stage Correction using a Cadaver Bone Allograft
}

\author{
by Al Kline, DPM ${ }^{1} \rrbracket$, Endolyn Garden, BS (Hons) ${ }^{2} \rrbracket$
}

The Foot and Ankle Online Journal 2 (5): 1

Brachymetatarsia is defined as congenital shortening of the metatarsal caused by premature closure of the epiphysis. The condition most commonly affects the fourth metatarsal of young and adolescent females. Correction of this deformity is either by callus distraction using an external fixator, or by one-stage surgical lengthening procedure using autogenous iliac bone graft. A case of brachymetatarsia is presented that is corrected by one-stage cadaver bone graft sterilized by the Biocleanse ${ }^{\circledR}$ method. Advantages include complete incorporation of the graft and healing characteristics similar to autogenic bone grafting without the need to harvest graft material.

Keywords: Brachymetatarsia, autogenous, allogenic, bone graft, allograft, biologics, Biocleanse ${ }^{\circledR}$ sterilization process

\author{
Accepted: April, 2009 \\ Published: May, 2009
}

This is an Open Access article distributed under the terms of the Creative Commons Attribution License. It permits unrestricted use, distribution, and reproduction in any medium, provided the original work is properly cited. ๑The Foot and Ankle Online Journal (www.faoj.org)

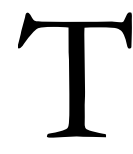
The exact etiology of brachymetatarsia is not known. It is thought to be either idiopathic congenital, acquired, or associated congenital. ${ }^{3}$ The idiopathic congenital etiology, which refers to the early closure of the epiphyseal plate, is thought to be the most common. The associated congenital etiology is accompanied by other conditions such as Albright's Syndrome or Down's Syndrome and parathyroid alterations. Acquired etiology refers to the early closure of the epiphyseal plate after suspected trauma. ${ }^{3}$

A variety of measurements have been described to define brachymetatarsia of the fourth metatarsal. ${ }^{2,3,4,7}$

Address correspondence to: $\mathrm{Al}$ Kline, DPM

3130 South Alameda, Corpus Christi, Texas 78404.

Email: al@kline.net

\footnotetext{
${ }^{1}$ Adjunct Clinical Faculty, Barry University School of Podiatric Medicine. Private practice, Chief of Podiatry, Doctors Regional Medical Center. Corpus Christi, Texas, 78411.

${ }^{2}$ Texas A\&M Graduate (Hons), Corpus Christi, Texas, Incoming first year student, Barry University School of Podiatric Medicine.
}

In 2003, brachymetatarsia was diagnosed "when one metatarsal ends $5 \mathrm{~mm}$ or more proximal to the parabolic arc".

In 2004, a morphofunctional study described a more specific measurement called the "angle of fourth metatarsal shortening or second-fourth angle" to quantify the definition of brachymetatarsia. ${ }^{3}$ Brachymetatarsia is defined as a second-fourth angle of less than 52.2 degrees in males and 50.5 degrees in females. ${ }^{3}$ Using both techniques, in our case report, the fourth metatarsal measured more than $5 \mathrm{~mm}$ of shortening from the parabolic line and the second-fourth angle is less than 50.5 degrees respectively ( 45.2 degrees). The parabolic difference is a $10 \mathrm{~mm}$ shortening. (See radiograph Fig. 2)

It has been reported that the fourth metatarsal is more likely to be affected by this condition, although many studies vary in their reports. ${ }^{3,40}$ The majority of cases are seen in females ( $98: 4$ female to male ratio respectively) and $72 \%$ of these cases occur in both feet. $^{4} \quad$ Brachymetapody, a noticeable shortening of the toe, can also present with brachymetatarsia. 


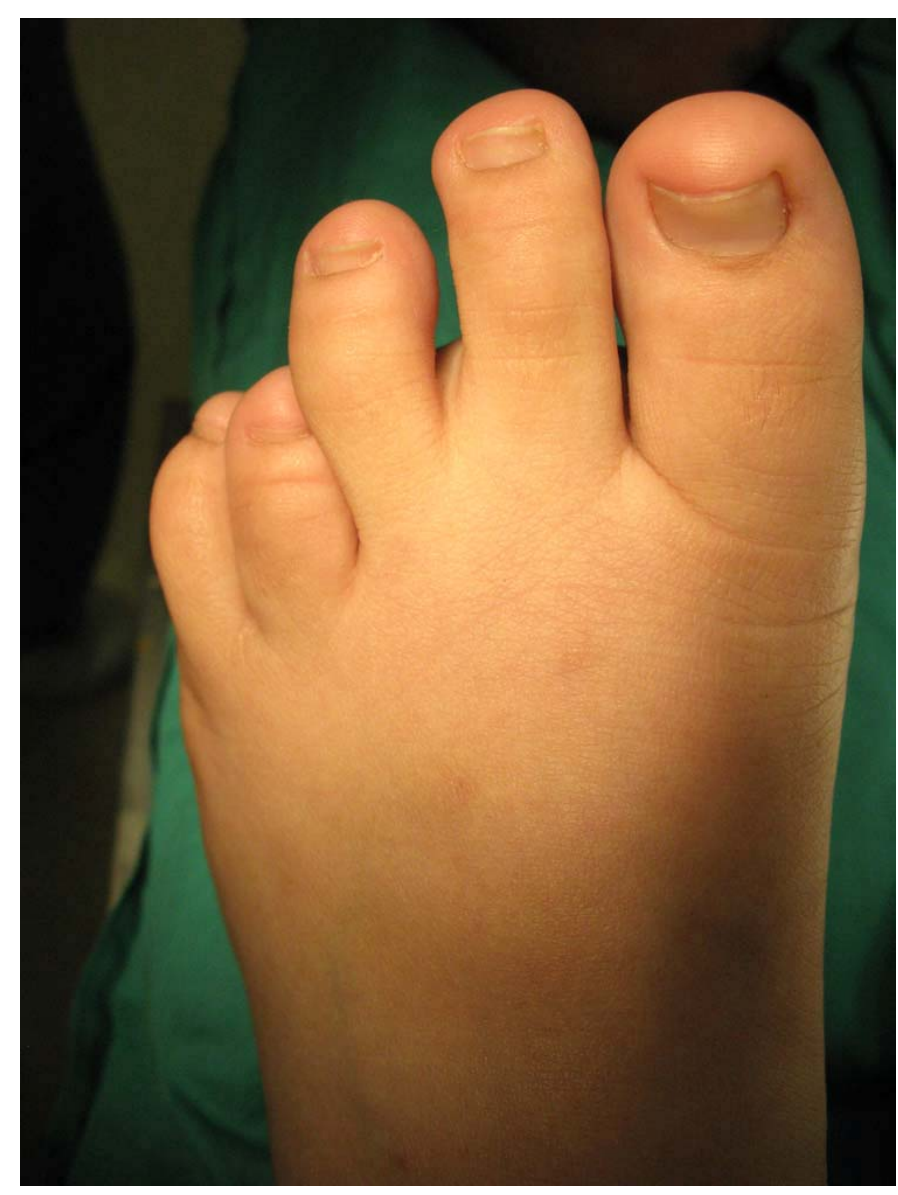

Figure 1 I nitial presentation of brachymetatarsia of the fourth metatarsal. Typically, the $4^{\text {th }}$ toe is short and contracted due to the immature growth and congenital shortening of the fourth metatarsal at 13 years age.

Conservative treatments include using metatarsal padding within the shoes. ${ }^{1}$ Digital padding and toe splinting may also be attempted. When this is ineffective, surgical correction may be indicated if the patient is experiencing metatarsalgia or have difficulty getting shoes to fit properly. These patients may develop painful calluses, or have a dislocated digit (also known as a "floating toe"). Many patients also express discontent with the appearance of the foot, but this alone is not usually an indication for surgery. ${ }^{1}$ However, having the deformity may cause the patient to be overly self-conscious which can lead to psychological issues such as depression. This is particularly important because the abnormality usually presents between the ages of 5 and 14 years in young females. ${ }^{4}$
When surgery is performed, the desired result is to relieve pain and restore functionality. ${ }^{5}$ The two methods used most often to correct this condition are gradual distraction using an external fixator, and onestage surgical lengthening using a bone graft. Gradual distraction involves surgically placing an external fixator on the metatarsal to be lengthened. One-stage lengthening of the metatarsal involves using allograft or autogenous bone, and interposing the graft within the metatarsal. When using an autogenous bone graft, the bone is harvested from the patient's own body and transplanted into another part of the body. A common site to harvest autogenous bone is the iliac crest. Other sites may also be used, such as the ribs, calcaneus and fibula. ${ }^{12}$ An allograft is "any tissue harvested from one individual and implanted into another of the same species ${ }^{13}$," and is used as a substitute for autogenous bone. The allograft bone used in this case were prepared by sterilization and disinfection methods that include gamma irradiation and a low temperature chemical sterilization method known as BioCleanse ${ }^{\circledR} .11$

\section{Case Report}

A 13 year old female presents to the office with pain and discomfort involving the left fourth toe. The toe 'rides up' on the foot and interferes while wearing closed shoes. (Fig.1)

Radiographic evaluation reveals a congenitally short fourth metatarsal. (Fig. $2 \mathrm{~A}$ and B) The patient tried modifying her footwear, but with limited success. At initial visit, the patient was fitted with a digital Budin type splint in an attempt to help plantarflex the digit and eliminate pain. We also initiated dorsal digital padding to protect the toe while in the shoe. Most conservative measures were inadequate and we opted to bring the patient to surgery in order to correct the brachymetatarsia and lengthen the toe.

The patient has an allergy to Suprax ${ }^{\circledR}$ and is taking Ibuprofen for pain and swelling. The patient has no medical conditions and is young and healthy. 


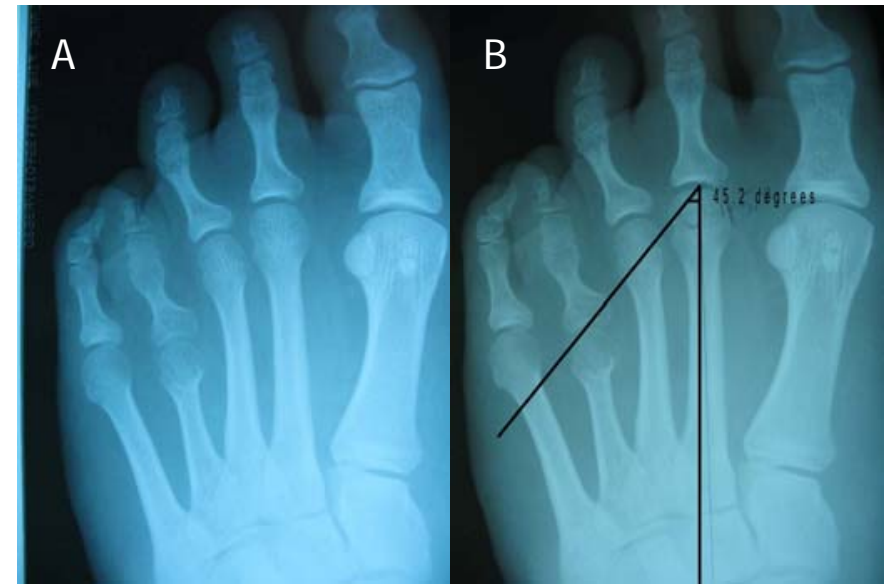

Figures 2A and 2B Radiographs show a typically short fourth metatarsal. Notice how the fifth toe has rotated in a digital varus orientation. All epiphyseal growth plates have already closed. (A) The second-fourth angle measured 45.2 degrees which significantly less than the normal parameter of 50.5 degrees (B)

Surgery was discussed with the patient and mother. We described the two options for surgery including callus distraction with an external fixator or a one stage procedure including inter-positional bone graft. Because of her age and health status, we opted for the one stage lengthening. We also described the various types of grafting techniques including autogenous bone grafting from the patient's own iliac crest to using allogenic, cadaver sterilized bone graft.

Complications of this procedure were discussed including failure of graft material, vascular compromise to the fourth toe and metatarsophalangeal joint stiffness. Two weeks before her surgery, she was asked to manually place distal traction on the toe every night for about 5 to 10 minutes daily.

\section{Surgical Technique}

The patient was brought to the operating room. Under sterile technique, a small linear incision was made along the dorsal mid-shaft region of the fourth metatarsal. The extensor tendon was identified and lengthened by standard z-plasty technique. (Figs. $3 \mathrm{~A}$ and B)

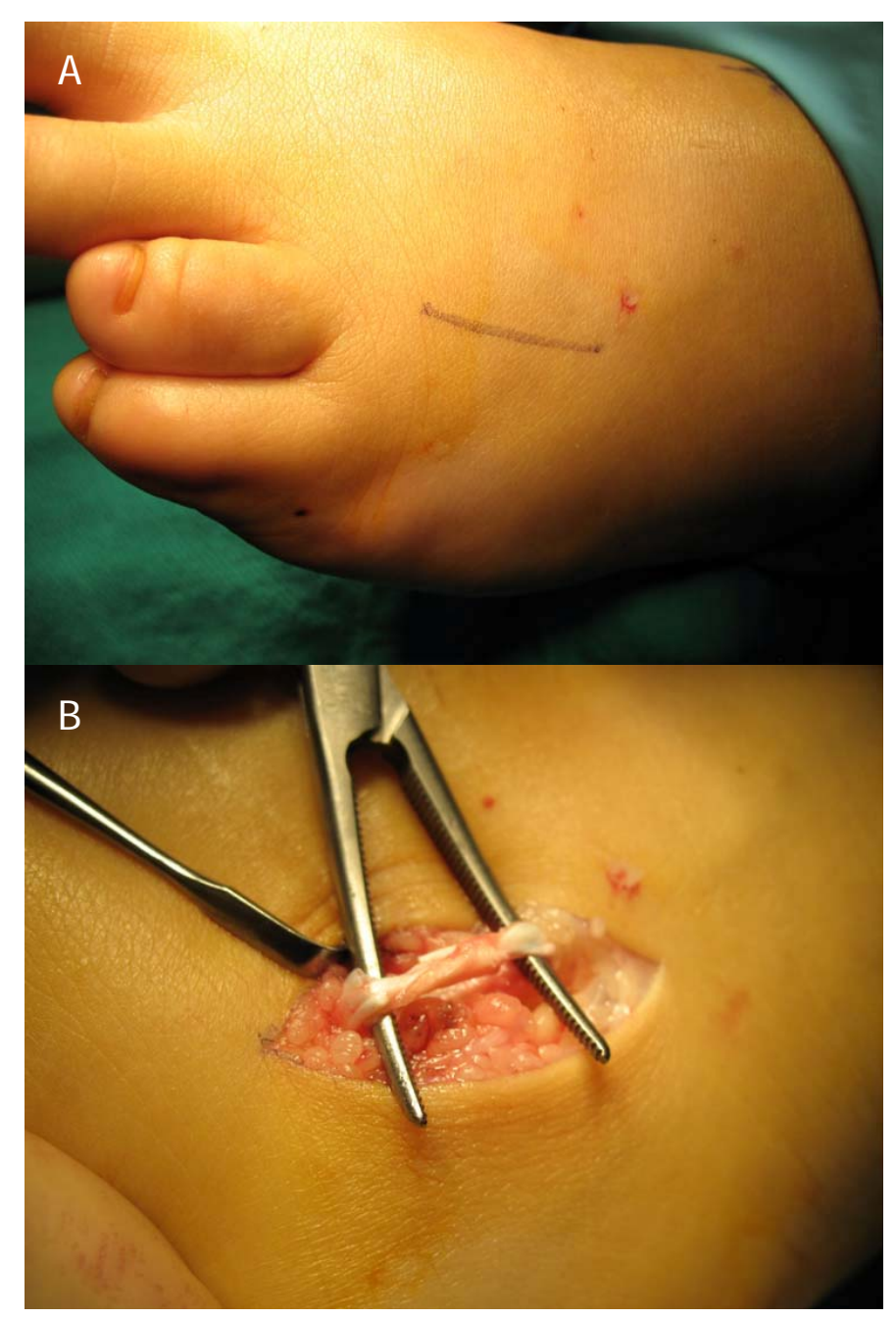

Figures $3 \mathbf{A}$ and $3 \mathbf{B}$ A small linear incision is oriented along the long axis of the fourth metatarsal. (A) An extensor z-slide tenotomy is performed to prevent dorsal contracture of the fourth toe during the metatarsal lengthening. ( $B$ )

Using blunt and sharp dissection technique, the midshaft region of the fourth metatarsal was identified. A small surgical bone saw was used to perform a transverse osteotomy through the metatarsal.

Using a laminar spreader, $2 \mathrm{~cm}$ of distraction is placed between the distal and proximal portions of the fourth metatarsal. It is important to gradually place increasing distraction stress through the metatarsal. (Figs. 4 A - C) 


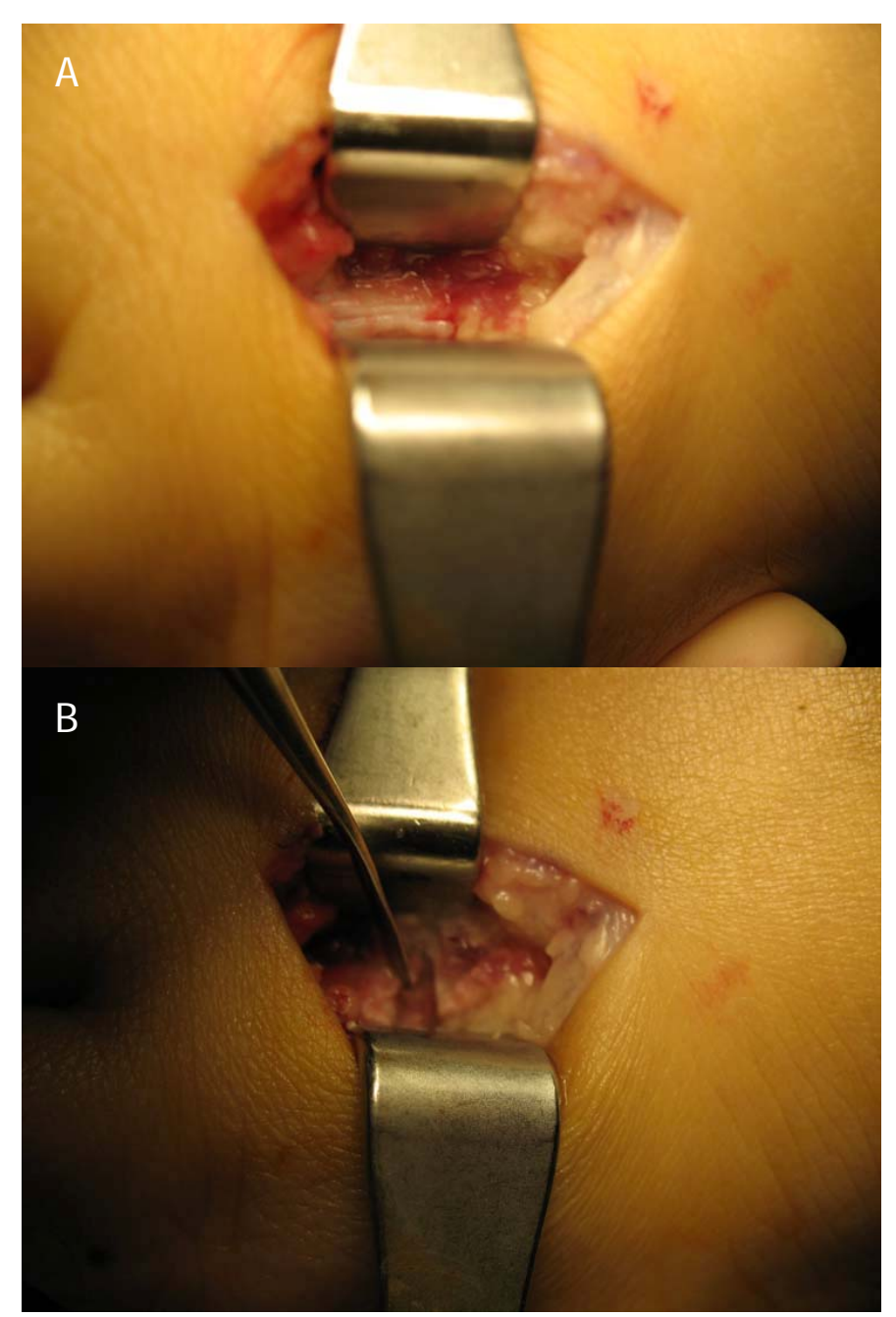

This allows for gradual lengthening of the neurovascular structures of the fourth toe and promotes a gradual plastic deformation of the tissues.

The metatarsal is gradually lengthened over 30 minutes to 1 hour. During this period, the graft can be shaped and prepared for implantation. It is important to realize that the fourth metatarsophalangeal joint will become inherently stiff and rigid during this process. The elastic properties of the surrounding tissues including the joint capsule will slowly begin to deform and relax. A too rapid distraction will cause soft tissue contracture leading to vascular spasm, so gradual distraction is recommended.

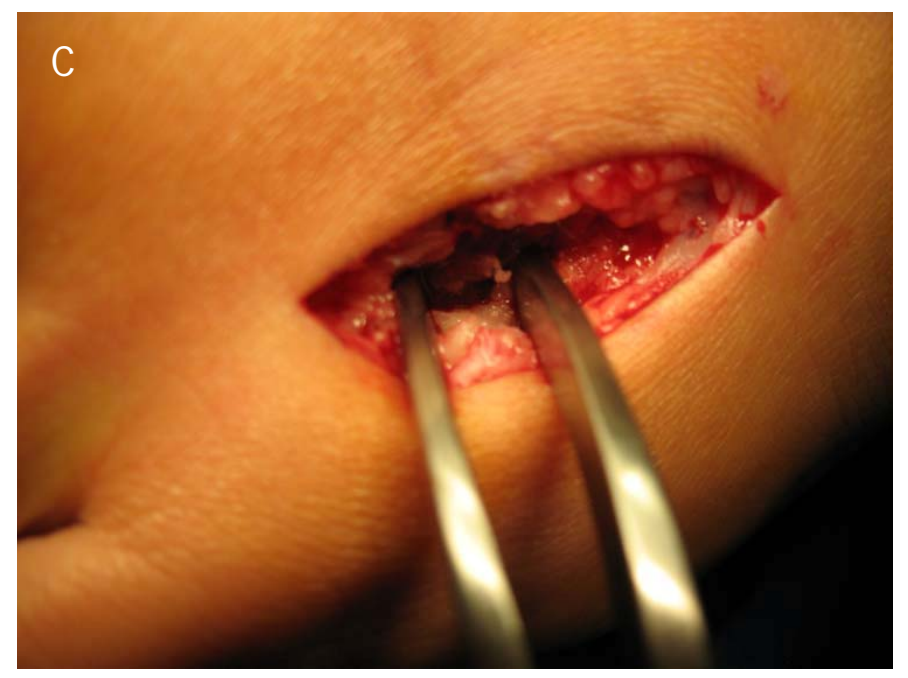

Figures 4A, 4B and 4C The mid-shaft region of the fourth metatarsal is exposed taking care to not strip the periosteum from each side of the bone. (A) A small bone saw is used to perform a transverse osteotomy perpendicular to the long axis of the fourth metatarsal. (B) A laminar spreader is then used to place a distractive force along the long axis of the fourth metatarsal. The laminar spreader is slowly spread apart over an hour to eliminate any incidence of vascular compromise. The metatarsophalangeal joint will become inherently stiff during the distraction process. Plastic deformation of the surrounding tissues is promoted by gradual stress distraction. (C)

During the hour of controlled distraction, the cadaver graft is prepared. It is important, when preparing the graft, that one take bone approximating the thickness and length of the metatarsal. We chose to use a humoral graft and cut a $20 \mathrm{~mm}$ section to approximate the size and shape of the metatarsal. Although the metatarsal gap measured and desired length is measured at about $10 \mathrm{~mm}$ on radiograph, a larger graft is recommended to be initially used. The graft is easier to handle and drill, then can be remodeled to a smaller size prior to insertion.

Once the graft is to the desired shape, a $.062 \mathrm{~mm}$ Kirschner wire is used to drill a hole along the long axis of the graft. This is called pre-drilling the graft. This graft was completely cortical. Pre-drilling the graft will allow for easier placement in the final stages of the operation. 


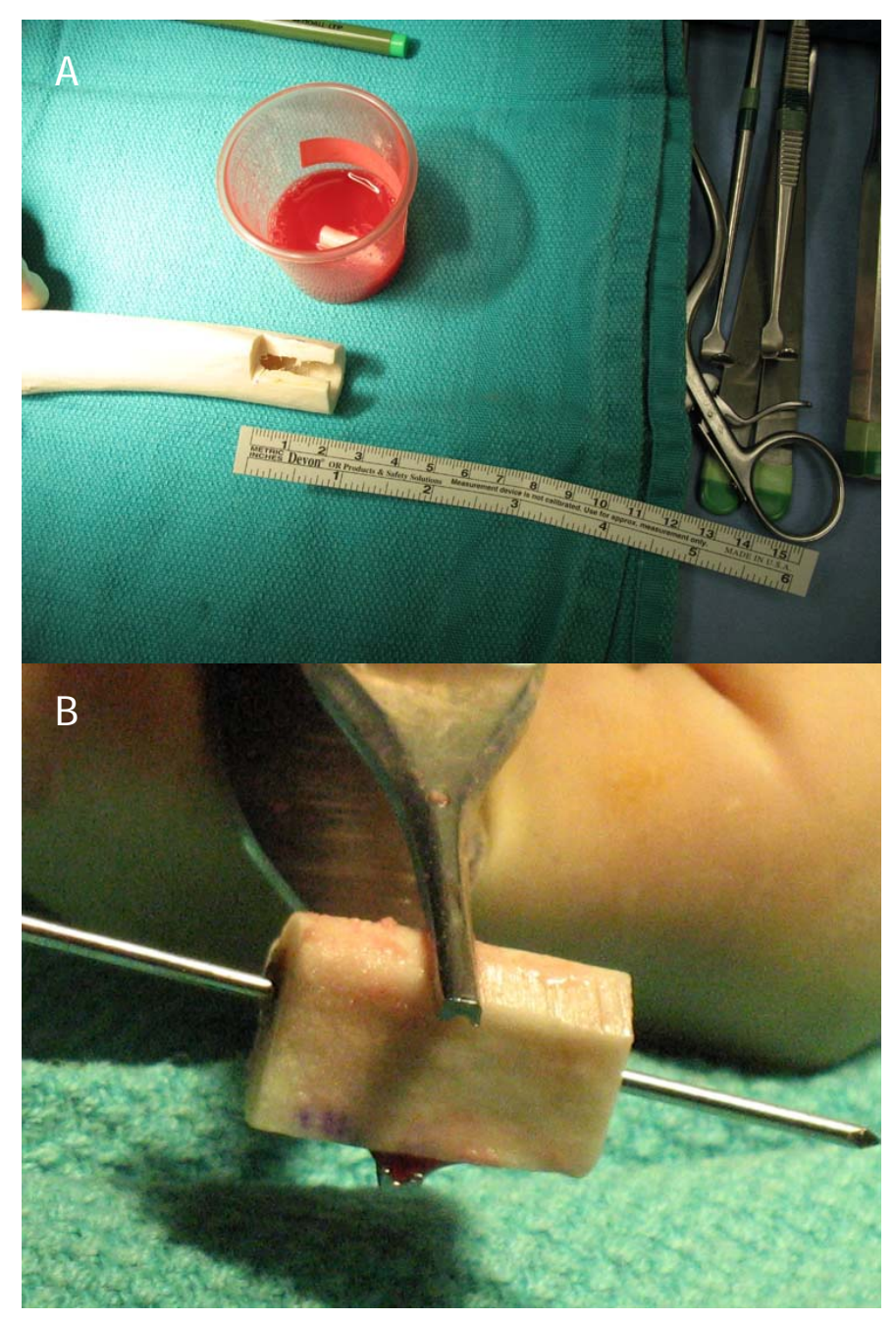

Once the graft is prepared, blood is drawn from the patient and a platelet and white blood cell concentrate is prepared and placed on the back table. The graft is then placed in the concentrate slurry while completing the distraction process. (Figs. $5 \mathrm{~A}-\mathrm{C}$ )

When the distraction process is complete, the graft is now ready for placement. This stage can be technically challenging due to the persist tightness of the confined space. The graft often has to be reshaped or slightly shortened for proper placement. That is why it is important to properly measure the distance of distraction prior to graft placement.

At this point the laminar spreader is removed and the wire is reverse drilled along the distal portion of the metatarsal through the digit. The bone must be angled and care must be taken to not plantarflex the digit too much once pinned.

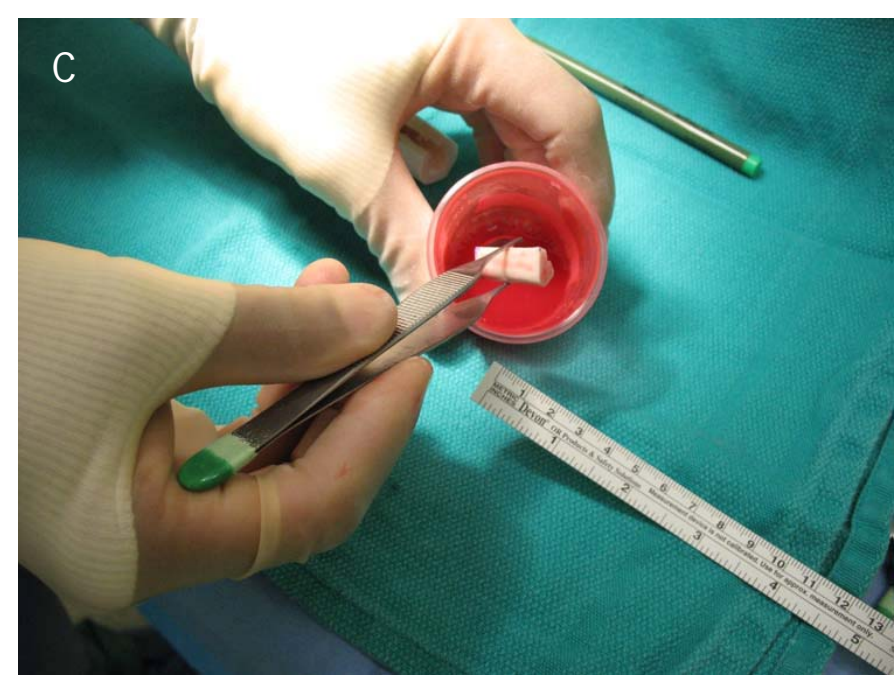

Figures 5A, 5B and 5C The allograft is cut directly from a hard cortical section of humeral cadaver bone. (A) Once the bone is shaped, the graft is pre-drilled along the long axis of the bone to prepare for interpositional insertion of the graft to the fourth metatarsal. (B) While the laminar spreader is distracting the bone, once the graft is ready, it can remain in the platelet and white blood cell concentrate taken from the patient's own blood. (Biomet ${ }^{\circledR}$ Bioorthologics GPS ${ }^{\circledR}$ III)

Once the k-wire is in proper alignment, the graft is carefully inserted within the metatarsal. The most challenging aspect of this surgery is aligning the predrilled hole with the k-wire and through-drilling to the most proximal segment of the fourth metatarsal. (Figs. 6 A-C)

Once the graft is in place, the remaining platelet and white blood cell slurry is lavaged into the wound prior to closure. A small Jergen's ${ }^{\circledR}$ ball is placed on the kwire and the foot is then dressed in a smile gauze dressing and placed non-weight bearing in a posterior leg splint. (Figs. 7 A - C)

After 2 weeks in a posterior splint and when the sutures are removed, the patient is placed in a short leg fiberglass cast for an additional 6 weeks. The entire immobilization period is about 8 weeks before partial to full weight bearing can resume. Radiographs performed at the end of 8 weeks reveals solid and complete incorporation of the graft along the metatarsal shaft. 


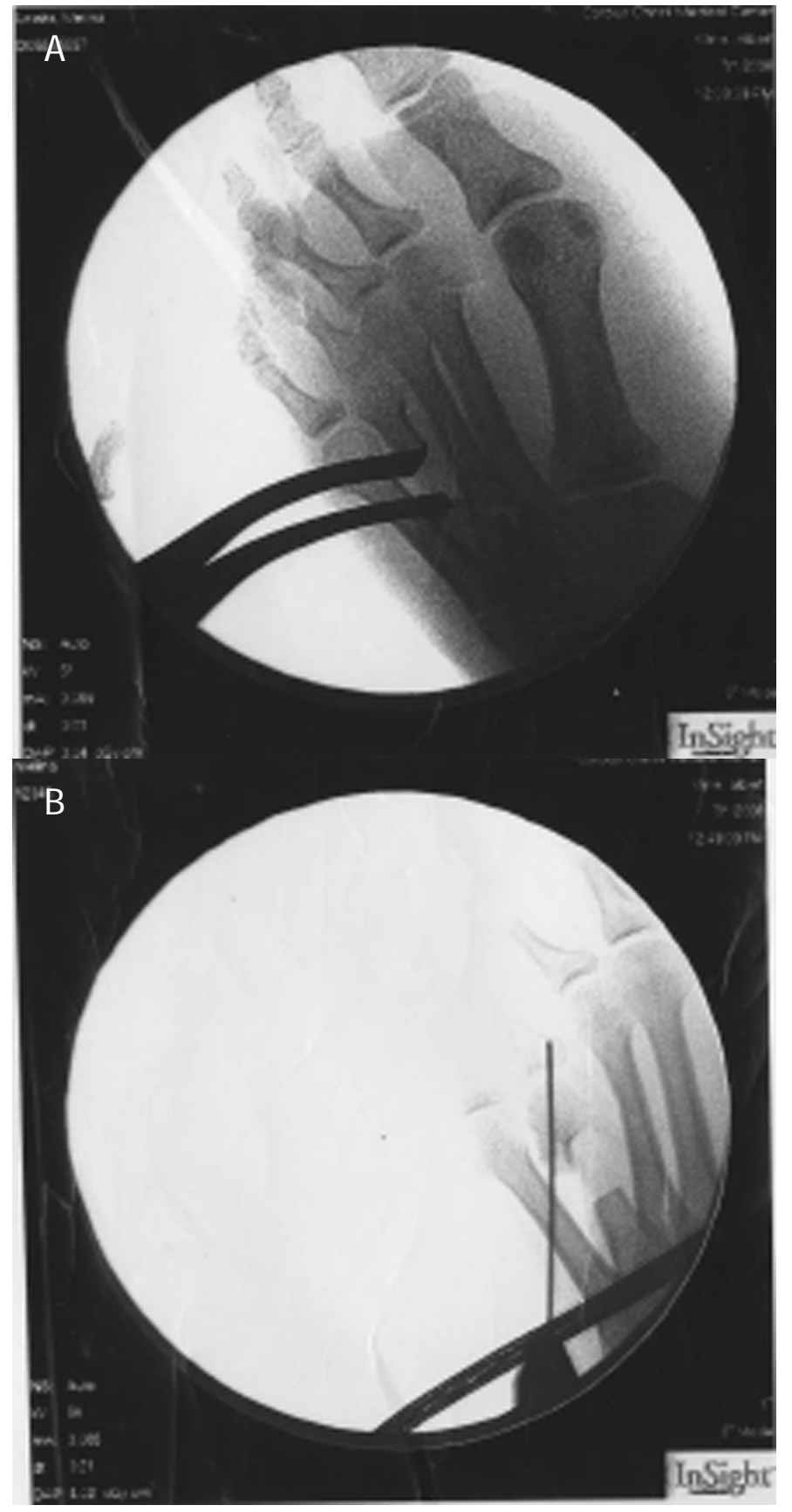

The patient has now been seen over the last year without pain or complication to the graft. The toe actually moves without any stiffness to the metatarsophalangeal joint on range of motion. She is very pleased with her surgical outcome. (Figs. 8 and 9)

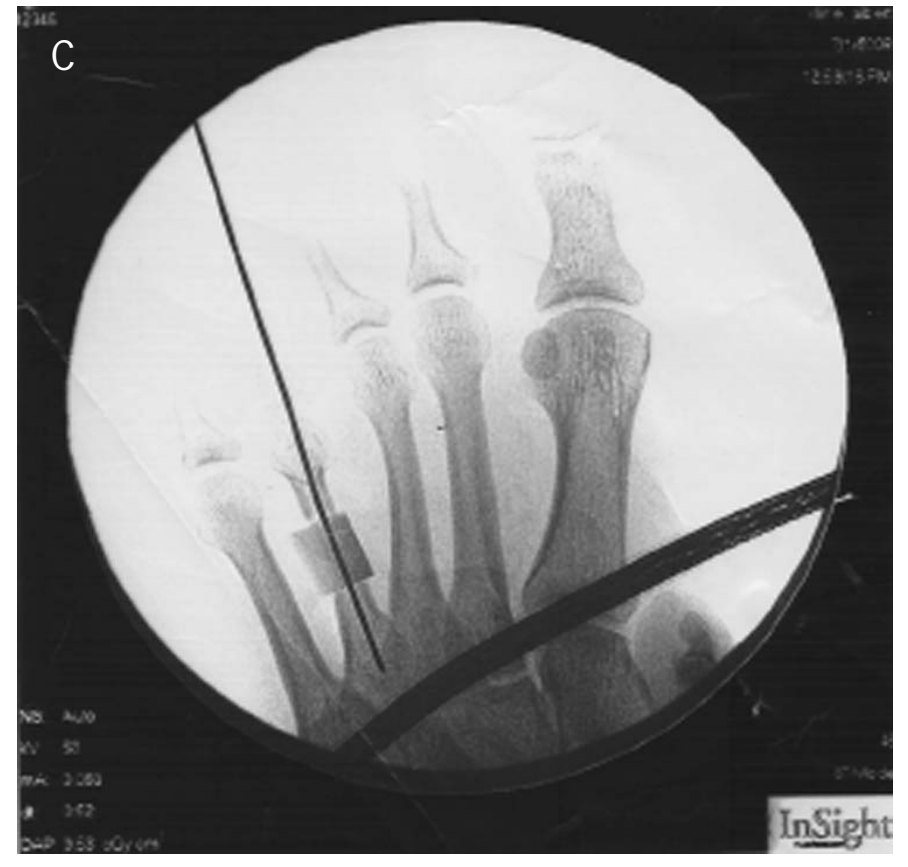

Figures 6A, 6B and 6C The laminar spreader is removed after an hour of distraction. The mini c-arm is used to determine the proper amount of distraction to attain the proper metatarsal length and parabola. (A) The $k$-wire is directed distally first. The bone is angulated and then drilled through the fourth digit. (B) The graft is then interposed within the fourth metatarsal, drilled and stabilized with a $.062 \mathrm{k}$-wire. (C)

\section{Discussion}

The author's have found the use of an allogenic bone graft to have the same characteristics and properties as autogenous bone in one-stage metatarsal lengthening procedures, but without the need to harvest bone graft from the patient. They both have osteogenic, osteoconductive and osteoinductive properties. ${ }^{18}$ Osteogenic properties refer to the properties that promote the synthesis of new bone. Osteoconductive properties are those properties of the graft that provide framework where the formation occurs. Finally, osteoinduction is the ability of the graft to "stimulate the host precursor cells to form new bone through differentiation into chondroblast or osteoblast". 18 One of our concerns before surgery was whether the graft would incorporate as normal bone. 


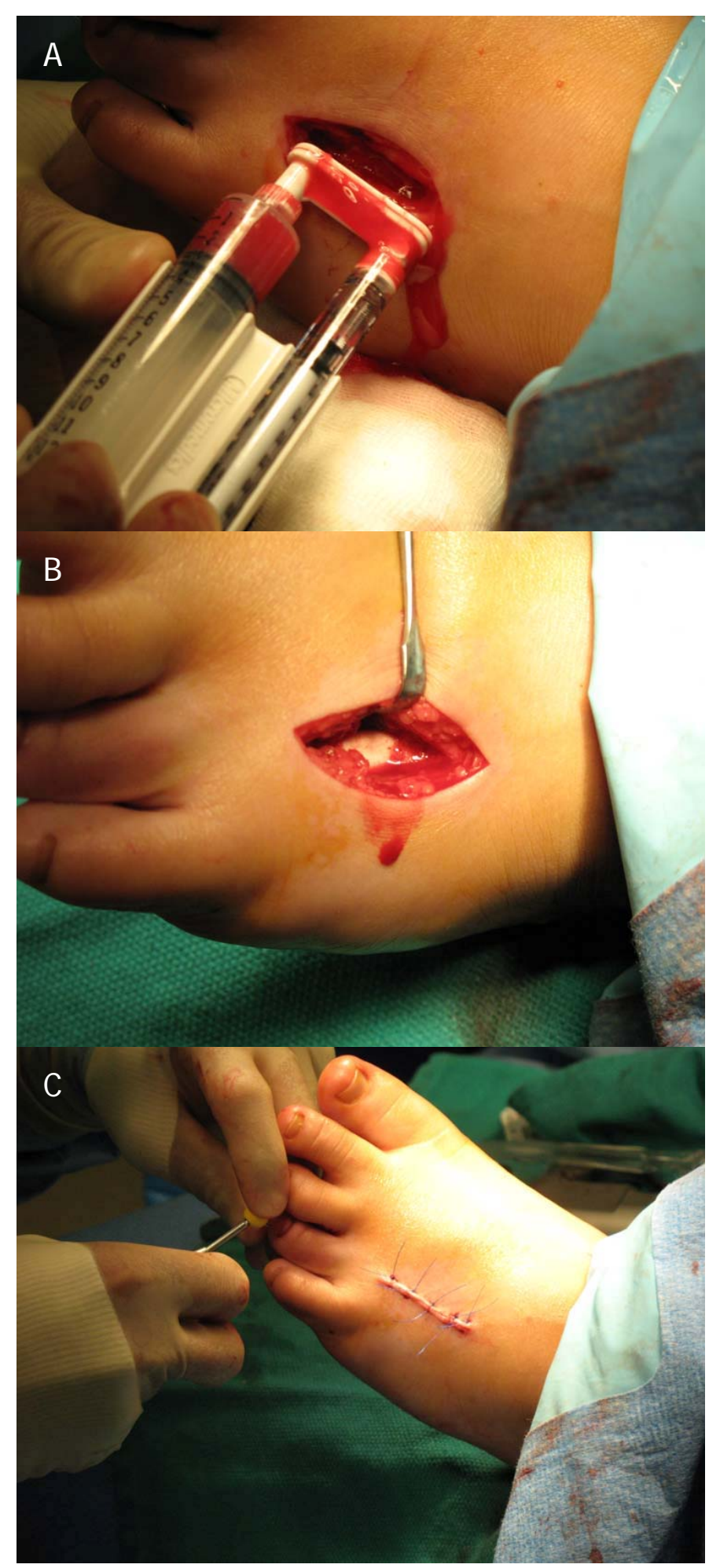

Figures 7A, 7B and 7C Once the graft is securely in place, the patient's own platelets and white blood cells are lavaged into the wound. (A and B) The pin is protected with a Jergen's $₫$ ball at the end of the k-wire and closed prior to application of dressings and a posterior leg splint. (C)
As previously mentioned, there are two techniques commonly used to treat brachymetatarsia: gradual distraction with external fixation and one-stage lengthening using bone grafts.

The first method involves applying an external fixator that is used to gradually lengthen the bone. This is achieved by surgically placing the fixator into the metatarsal that is to be lengthened. About a week post-operatively, the lengthening begins at a rate of $1 / 4$ mm four times per day for a total of $1 \mathrm{~mm}$ per day. ${ }^{2}$ This may take place over a period of several weeks. ${ }^{5}$ After the desired result is achieved, the fixator remains static for twice the amount of time it took to perform the distraction, during which time the patient remains non weight-bearing. ${ }^{5}$ The patient typically can tolerate full weight bearing once the fixator is removed. ${ }^{2}$ The reported advantage to this technique is the soft tissues and neurovascular structures are lengthened at the same time the bone is being lengthened. This tends to maximize the ability of the metatarsal to lengthen. ${ }^{2}$

There are complications that could arise when using gradual distraction over a longer period, but this appears to be more associated with the external fixator. Some of these include hyper-pigmentation around pin sites, pain during distraction, stiffness, decreased range of motion, scarring, deformities, joint dislocation, prolonged bone consolidation and pintrack infections. ${ }^{1,2,5,8}$

One-stage lengthening is a process where autogenous or allograft bone is grafted to lengthen the metatarsal. The advantages of this procedure include a shorter bone consolidation period, smaller incision, and less morbidity. ${ }^{8,10}$ Some of the disadvantages and complications involved with autogenous bone grafting include technical difficulty, neurovascular damage, small gain in length, and donor site morbidity. ${ }^{8,10}$

It appears that gradual lengthening in the operating room using a laminar spreading and applying distraction stress gradually over 30 minutes to 1 hour will not cause vascular compromise. 


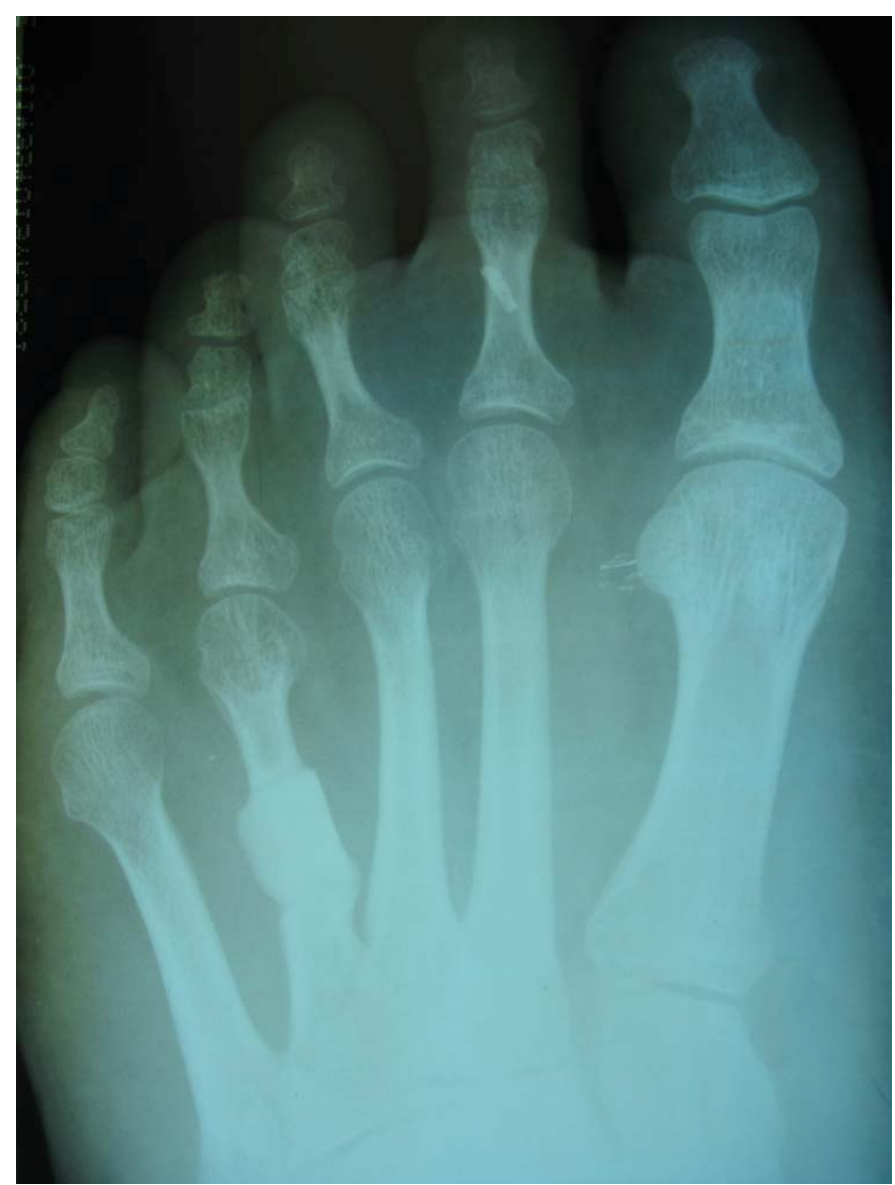

Figure 8 At 8 weeks, the bone graft shows signs of bone interposition and callusing. Deformation stress is noted along the proximal half of the $4^{\text {th }}$ metatarsal, but does not compromise the overall shape of the metatarsal.

A number of studies have been reported on the viscoelastic properties of the surrounding soft tissues during metatarsal lengthening. ${ }^{4,10}$ Stress relaxation will promote a lengthening of soft tissue when gradually performed, even in a relatively short period of time. A too rapid distraction of surrounding tissues will cause more contracture and vascular spasm with tissue compromise. Using this gradual distraction technique with a simple laminar spreader, we were able to achieve over $10 \mathrm{~mm}$ of lengthening within an hour without vascular compromise to the toe.

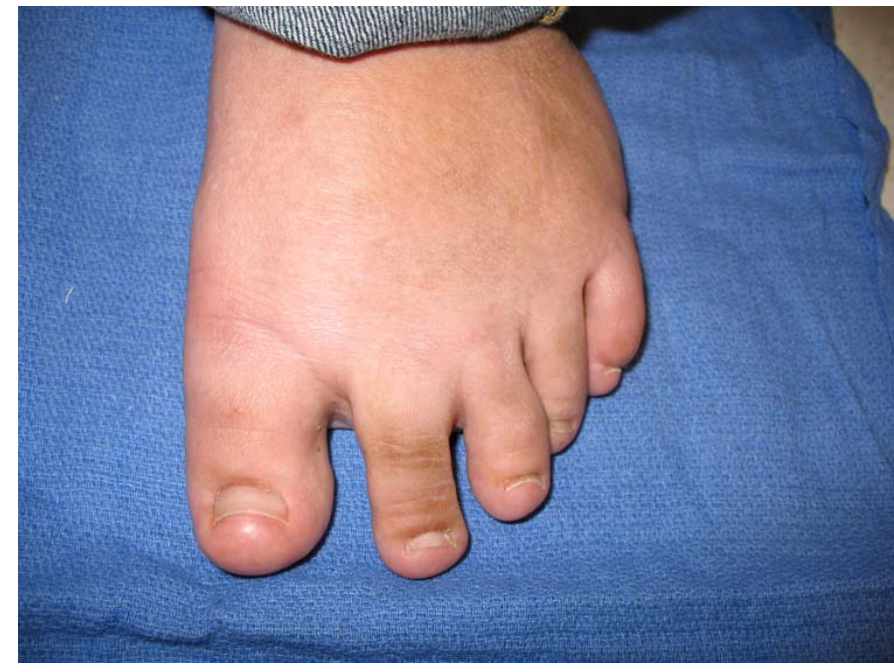

Figure 9 After 6 months, the patient is very pleased with the restoration of metatarsal and toe length. There is excellent fourth metatarsophalangeal joint range of motion without pain or discomfort.

Allogenic and autogenous bone grafts have similar properties including bone healing characteristics and incorporation rates. The process of bone healing occurs in four stages; inflammation, soft callus formation, hard callus formation and bone remodeling. During the first stage, there is bleeding at the site which results in a hematoma. Inflammatory cells then penetrate the hematoma to fight infection, secrete cytokines and growth factors and promote clotting. In the next stage chondrocytes and fibroblast produce a soft callus to provide mechanical support and a template for the bony callus. The third stage is where most of the osteogenesis occurs. There is a high level of osteoblastic activity and formation of mineralized bone. The soft callus is slowly removed and revascularization occurs. ${ }^{20}$ In the final stage, remodeling of the bone occurs, blood circulation to the area improves and the bone becomes compact. Complete bone healing takes $6-8$ weeks, although factors such as movement, smoking, poor nutrition, age and disease can affect the healing rate. ${ }^{21}$ 
An advantage to allogenic bone grafting is that there is no need to harvest bone from the patient, thus there is no donor site and a second surgery site. Having a second surgery site, or in this case, a donor site can potentially make surgery more complicated, and increase the risk of infection as well as creating increased pain along the donor site. It is very common for the donor site to be more painful after surgery than the recipient site, especially at the iliac crest. With allogenic bone, there is no donor site pain, no type matching or rejection, and the allogenic bone can be pre-shaped to decrease the surgery time. ${ }^{16}$ To our knowledge, this is the first reported successful correction of brachymetatarsia with complete incorporation of a cadaver allograft using the Biocleanse ${ }^{\circledR}$ sterilization process.

\section{The BioCleanse ${ }^{\circledR}$ Sterilization Process}

The BioCleanse ${ }^{\circledR}$ sterilization process is used by Regeneration Technologies to prepare allograft tissue for surgical uses. These implants are used in spinal, sports medicine, general orthopedic, cardiovascular, and dental surgeries. ${ }^{17}$ Before any tissue is used, a medical and social history of the donor is obtained from the donor's family. The tissue is then inspected and screened for diseases (such as HIV and hepatitis). ${ }^{15}$ Upon approval, the tissue enters into the automated sterilization process. ${ }^{14}$ In the first step of the sterilization process blood, lipids, and marrow are removed from the bone via a vacuum/pressure process to reduce the risk of immune response in the recipient. Next, chemical sterilants are used to eliminate pathogens. This process is designed to go deep within the tissue matrices to eliminate pathogens such as bacteria, viruses, and fungi. Finally, the germicides are removed, and the tissue's biocompatibility is preserved in the process. ${ }^{13}$ In order to ensure a low contamination rate, surface sterilization is incorporated during final packaging through low doses of gamma irradiation or hydrogen peroxide gas plasma. ${ }^{13}$
Mroz, et al., in analyzing the biomechanical properties of allograft bone treated by the sterilization process concluded "Sterilization of allograft bone with Biocleanse ${ }^{\circledR}$ does not significantly alter the mechanical properties when compared with untreated samples. The effect of this sterilization process on the osteoconductive and osteoinductive properties of allograft bone must be determined.',22

In this case report, it appears the allograft incorporated well within the surrounding bone and tissue and provided this patient with adequate bone lengthening without the need for autogenous bone harvest.

\section{References}

1. Gilbody J, Nayagum S: Lengthening of the first metatarsal through an arthrodesis site for treatment of brachymetatarsia: A case report. Journal of Foot Ankle Surgery. 47 (6): 559 - 564, 2008.

2. Houshian S, Skov O, Weeth R: Correction of congenital brachymetatarsia by gradual callus distraction. Scand J of Plast Reconstr Surg Hand Surg. 36373 - 375, 2001.

3. Pedro V. Munuera Martínez PVM, Guillermo Lafuente Sotillos G, Domínguez Maldonado G, Luis Salcini Macías J, Martínez Camuña L: Morphofunctional Study of

Brachymetatarsia of the Fourth Metatarsal. J Am Podiatric Assoc . 94 (4) 347-352, July-Aug. 2004.

4. Goforth W. et al Brachymetatarsia of the Third and Fourth Metatarsals. J Am Podiatric Assoc. 91 (7) 373-378, 2001.

5. Wilusz P, Van P, Pupp GR: Complications associated with distraction osteogenesis for the correction of brachymetatarsia: A review of five procedures. J Am Podiatric Assoc. 97 (3) 189 194, 2007.

6. Allmendinger A, Yeghiayan P, Perone R, St. Vincent's Medical Center in New York City: Case of the month. diagnostic imaging 31( 1) [online] Accessed February 7, 2009.

7. Kim HT, Lee SH, Yoo CI, Kang JH, Suh JT: The

Management of Brachymetatarsia. Journal of Bone Joint Surgery. 85B (5) $661-665,2003$.

8. Kim J, Baek GH, Chung MS, Yoon PW: Multiple Congenital Brachymetatarsia: One-Stage Shortening and Lengthening Procedure Without Iliac Bone Graft. Journal of Bone Joint Surgery. 86B (7): 1013 - 1015, 2004.

9. Bone Graft Alternatives. North American Spine Society [online] Assessed February 15, 2009.

10. Baek GH, Chung MS: The Treatment of Congenital Brachymetatarsia by One-Stage Lengthening. Journal of Bone Joint Surgery. 80B (6): 1040 - 1044, 1998. 
11. (2003) BioCleanse Tissue Sterilization :Sterilization Validation. Regeneration Technologies, Inc. [online] Accessed March 21, 2009

12. Finkemeier C. Bone-Grafting and Bone-Graft Substitutes. Journal of Bone Joint Surgery. 84A (3): 454-463, 2002.

13. Bostrom MPG, Seigerman DA: The Clinical Use of Allografts, Demineralized Bone Matrices, Synthetic Bone Graft Substitutes and Osteoinductive Growth Factors: A Survey Study. Hospital for Special Surgery, 2005 [online] Accessed March 21, 2009.

14. No author: A New Standard for Tissue Sterility.

Regeneration Technologies (2006) [online] Accessed March 21, 2009.

15. No author: From Donation to Implantation. Regeneration Technologies (2006) [online] Accessed March 21, 2009.

16. No author: Biologics vs. other materials. Regeneration Technologies (2006) [online] Accessed March 21, 2009.

17. No author: Biologics Implants. Regeneration Technologies (2006) [online] Assessed March 21, 2009.

18. Sen MK, Miclau T: Autologous Iliac Crest Bone Graft:

Should it Still be the Gold Standard for Treating Nonunions?. Injury. 38 (1) S75-S80 2007.

19. Malay DA: Closer look at bone graft substitutes. Podiatry Today (18) 1 Sept, 2005 [online] Accessed April 15, 2009

20. Schindeler A, et al Bone Remodeling During Fracture Repair: The Cellular Picture. Seminars in Cell \& Developmental Biology 19: 459 - 466, 2008

21. No author: Bone Healing (2008, May 26). American College of Foot and Ankle Surgeons [online] Accessed April 15, 2009.

22. Mroz TE, Lin EL, Summit MC, Bianchi JR, Keesling JE Jr, Roberts M, Vangsness CT Jr, Wang JC: Biomechanical analysis of allograft bone treated by novel tissue sterilization process.

Spine Journal 6 (1): 34 - 39, 2006. 